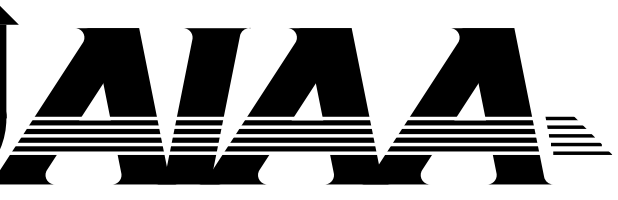

AIAA 2003-3532

Analysis of Extensive Cross-Flow Separation using Higher-Order RANS Closure Models

J. H. Morrison

A. G. Panaras

T. B. Gatski

G. A. Georgantopoulos

21st AIAA Applied Aerodynamics Conference

June 23-26, 2003

Orlando, FL 
AIAA-2003-3532

\title{
Analysis of Extensive Cross-Flow Separation using Higher-Order RANS Closure Models
}

\author{
J. H. Morrison * $\quad$ A. G. Panaras ${ }^{\dagger} \quad$ T. B. Gatski ${ }^{\ddagger} \quad$ G. A. Georgantopoulos $\S$
}

The turbulent flow fields associated with the incompressible flow over a 6:1 prolate spheroid at high angle of attack, and the supersonic flow over an ogive cylinder are studied. Both these flows are characterized by large separation and vortical flow regions and therefore provide a challenging database for comparison of turbulent closure models. Of interest is the ability to predict the effects of separation and associated vortical motion common to both flows. Two turbulent models are investigated that each represent the class of linear eddy-viscosity models (LEVMs) and explicit algebraic stress models (EASMs). Since the EASM accounts for anisotropic effects, the influence of these effects on flow field predictions can be assessed. The EASM model is shown to both improve the separation location prediction and pressure trough under the secondary vortex on the $6: 1$ prolate spheroid at high angle of attack and high Reynolds number, and improve the prediction of the separation location on a supersonic ogive cylinder.

\section{Introduction}

Streamwise, or longitudinal, vortices are formed in various types of three-dimensional separated flows. The high-incidence flow about a slender body is the most widely known case. Crossflow separation occurs when fluid flowing circumferentially from the windward to the leeward side of such a body separates from the sides of the body along a separation line roughly parallel to its longitudinal axis. The fluid rolls up and forms two primary vortices on the leeward side on both sides of the symmetry plane. The extent of the crossflow and the strength of the vortices grow as the angle of attack is increased. If the strength of the primary vortices is sufficiently large, secondary separation is induced below the primary ones, even if the boundary layer is turbulent. A similar type of separation is established in the highincidence flow about delta wings, as well as in the flow

\footnotetext{
* Senior Member AIAA, Research Scientist, Computational Modeling and Simulation Branch, Aerodynamics, Aerothermodynamics and Acoustics Competency, NASA Langley Research Center, Hampton VA 23681

†Associate Fellow AIAA, Consulting Engineer, Agias Elenis 63, Athens 157 72, Greece

$\ddagger$ Senior Research Scientist, Computational Modeling and Simulation Branch, Aerodynamics, Aerothermodynamics and Acoustics Competency, NASA Langley Research Center, Hampton VA 23681

$\S$ Professor, Laboratory of Aerodynamics, Hellenic Air Force Academy, Athens, Greece

This paper is a work of the U.S. Government and is not subject to copyright protection in the United States.
}

about various elements of high-speed vehicles (inlets, wing- and tail-fuselage junctures), where swept shockwaves interact with boundary layers and induce the appearance of quasi-conical separation vortices.

Though the numerical simulations of flows with extensive separation have significantly contributed to the understanding of the nature of these flows, the accuracy of the predictions has not been exceptionally high. In various Navier-Stokes calculations, that employ algebraic turbulence models and two-equation models, it has been found that the computations agree well with the data for moderate interaction strengths, but systematically underpredict the size of the interaction domain with increasing interaction strength. For example, in the case of high angle of attack flows, Panaras ${ }^{1}$ reviewed calculations on a 6:1 prolate spheroid using algebraic and half-equation turbulence models. The review showed that algebraic models with appropriate modifications could predict surface pressures at high angles of attack relatively well; however, these algebraic models required user intervention and were not suitable for complex flows. This ad hoc modification of the algebraic turbulence model had the effect of reducing the eddy-viscosity coefficient in the region of crossflow separation. The same technique also proved successful in the case of swept shock turbulent boundary layer interactions. ${ }^{2}$ A rationale for the success of these modified turbulence models that provide reduced turbulent stress levels within the separation vortices is easily provided. ${ }^{3}$ When extensive cross-flow separation exists, the inner, turbulent part of the separated boundary layer winds around the core of the separation vortex. The outer layers, which are composed of low turbulence fluid, fold over the vortex and at the attachment region, penetrate into the separation bubble forming a low turbulence region that lies along the wall, underneath the vortex. The incorporation of this dynamic feature into a lower-order algebraic model, by replacing the equation of calculation of the eddy-viscosity coefficient in the region of separation by a new one, which yields small values when the velocity gradients are high, brings considerable improvement to swept shock/boundary layer interactions, ${ }^{4}$ crossing shocks and high angle of attack flows about slender bodies. ${ }^{5}$

Unfortunately, algebraic turbulence models suffer from their dependence on the definition of the length scale and are not viable for complex geometries. Higherorder turbulence models based on transport equations can account, in principle, for such transport effects as well 
as turbulence anisotropies that algebraic models cannot. With this motivation, the performance of two-equation and explicit algebraic Reynolds stress models are evaluated, using two high angle-of-attack flows with extensive cross-flow separation: an incompressible 6:1 prolate spheroid and a supersonic ogive cylinder.

A variety of wind tunnel tests have been carried out for a 6:1 prolate spheroid at DLR in Göttingen. The majority of the tests were carried out at moderate Reynolds numbers, but some measurements were also conducted in collaboration with ONERA at higher Reynolds numbers. Ahn and Simpson ${ }^{6}$ documented boundary layer transition and separation characteristics for the 6:1 prolate spheroid for a range of Reynolds numbers and anglesof-attack. Chesnakas and Simpson ${ }^{7}$ used an innovative, internally mounted LDV probe to measure the complete velocity vector and the Reynolds stress tensor from the viscous sublayer to the boundary layer edge at $R e=$ $4.2 \times 10^{6}$.

The review by Panaras ${ }^{1}$ of calculations on a $6: 1$ prolate spheroid were related to the DLR experiments. Tsai and Whitney ${ }^{8}$ analyzed the prolate spheroid using a high Reynolds number $K-\varepsilon$ model with wall functions. Their results reproduced the flow structure and showed good agreement with measured pressure data at low Reynolds number conditions ( $R e=4.2 \times 10^{6}$ ), but consistently under-predicted the measured windward pressure values. Kim et al. ${ }^{9}$ investigated the prolate spheroid using twoequation and differential Reynolds stress models. For the low Reynolds number case $\left(R e=4.2 \times 10^{6}\right)$ they matched the flow structure of the VPI results. However, the models that predicted the leeward pressures well typically underpredicted the windward pressures, and the models that predicted the windward pressures well typically overpredicted the leeward pressures.

Constantinescu et al. ${ }^{10}$ investigated the $6: 1$ prolate spheroid with both Reynolds averaged Navier-Stokes (RANS) and a detached eddy simulation (DES). DES is a hybrid approach that combines a RANS approach for near wall thin shear layers with a large eddy simulation (LES) of large scale turbulent structures. They pointed out that the boundary layer separation prediction is determined by the RANS model component of the DES procedure. They found that there were not substantial differences in the predicted values of wall pressure and skin friction between the RANS and DES approaches.

An experimental study of the flow around a 3-caliber tangent ogive-cylinder in a supersonic flow was conducted at ONERA to develop an experimental database for code validations. ${ }^{11}$ Panaras ${ }^{5}$ investigated both these test cases with an algebraic turbulence model. The results at $30^{\circ}$ angle-of-attack for the prolate spheroid match the experimental wall pressure distributions very well. In the case of the supersonic ogive cylinder, the agreement at $20^{\circ}$ angle-of-attack was better than that at the lower angle $\left(10^{\circ}\right)$. These results indicated that additional validation studies were warranted to further evaluate the predictive performance of turbulence models in such high speed flows.

\section{Numerical Method and Turbulence Models}

The compressible Reynolds averaged equations for the conservation of mass and momentum can be written using Favre-averaged variables as:

$$
\begin{gathered}
\frac{\partial}{\partial t}(\rho)+\frac{\partial}{\partial x_{j}}\left(\rho u_{j}\right)=0 \\
\frac{\partial}{\partial t}\left(\rho u_{i}\right)+\frac{\partial}{\partial x_{j}}\left(\rho u_{i} u_{j}+p \delta_{i j}\right)=\frac{\partial}{\partial x_{j}} \rho \sigma_{i j}-\frac{\partial}{\partial x_{j}} \rho \tau_{i j}
\end{gathered}
$$

where $\rho$ is the density, $u_{i}$ are the velocity components in the $x_{i}$ coordinate directions, $\mu$ is the molecular viscosity and $\rho \sigma_{i j}$ is the mean stress tensor given by

$$
\rho \sigma_{i j}=\mu\left[\left(\frac{\partial u_{i}}{\partial x_{j}}+\frac{\partial u_{j}}{\partial x_{i}}\right)-\frac{2}{3} \frac{\partial u_{k}}{\partial x_{k}} \delta_{i j}\right]
$$

The Reynolds stress tensor, $\rho \tau_{i j}$, is a product of the averaging process and must be modeled to close the system of equations.

\section{Turbulence Models}

Chesnakas and Simpson ${ }^{7}$ used a unique, internally mounted LDV system to measure the boundary layer on the 6:1 prolate spheroid. Their data showed that the flow gradient angle differs from the shear stress angle over most of the boundary layer. Linear eddy viscosity models assume that the shear stress angle and the mean flow angle are the same.

Rumsey and Gatski ${ }^{12}$ investigated the effect of $K$ $\varepsilon$ and $K-\omega$ forms of two-equation models on multielement airfoil. They demonstrated that models based on the $K-\varepsilon$ formulation were typically deficient in predicting adverse pressure gradient flows. Models based on the $K-\omega$ formulation provided a better prediction of adverse pressure gradient flows. Therefore, two models based on the $K-\omega$ formulation with and without non-linear terms are investigated in this study; the $K-\omega$ linear eddy viscosity model of Wilcox ${ }^{13}$ and the explicit algebraic stress model of Rumsey and Gatski. ${ }^{12}$

\section{$K-\omega$ Eddy Viscosity Model}

The $K-\omega$ model of Wilcox ${ }^{13}$ is a linear eddy viscosity model that relates the Reynolds shear stress tensor to the mean strain rate tensor using the Boussinesq assumption:

$$
-\rho \tau_{i j}=\mu_{t}\left(S_{i j}-\frac{1}{3} S_{k k} \delta_{i j}\right)-\frac{2}{3} \rho K \delta_{i j}
$$


where the strain rate tensor is given by

$$
S_{i j}=\frac{1}{2}\left(\frac{\partial u_{i}}{\partial x_{j}}+\frac{\partial u_{j}}{\partial x_{i}}\right)
$$

The eddy viscosity, $\mu_{t}$, is related to mean and turbulent quantities by

$$
\mu_{t}=C_{\mu} \frac{\rho K}{\omega}
$$

where $K$ is the turbulent kinetic energy and $\omega$ is the specific dissipation rate.

The turbulent kinetic energy and specific dissipation rate are calculated by solving the following transport equations:

$$
\begin{aligned}
\frac{\partial}{\partial t}(\rho K)+\frac{\partial}{\partial x_{k}}\left(\rho u_{k} K\right)= & \rho \mathcal{P}-C_{K 2} \rho \omega K+ \\
& \frac{\partial}{\partial x_{k}}\left[\left(\mu+\frac{\mu_{t}}{\sigma_{K}}\right) \frac{\partial K}{\partial x_{k}}\right] \\
\frac{\partial}{\partial t}(\rho \omega)+\frac{\partial}{\partial x_{k}}\left(\rho u_{k} \omega\right)= & C_{\omega 1} \frac{\rho \omega}{K} \mathcal{P}-C_{\omega 2} \rho \omega^{2}+ \\
& \frac{\partial}{\partial x_{k}}\left[\left(\mu+\frac{\mu_{t}}{\sigma_{\omega}}\right) \frac{\partial \omega}{\partial x_{k}}\right]
\end{aligned}
$$

where the production is given as

$$
\mathcal{P}=-\tau_{i j} \frac{\partial u_{i}}{\partial x_{j}}
$$

and $C_{\mu}=1.0, C_{K 2}=0.09, C_{\omega 1}=5 / 9, C_{\omega 2}=3 / 40$, $\sigma_{K}=2.0$, and $\sigma_{\omega}=2.0$.

\section{Explicit Algebraic Stress Model}

The explicit algebraic stress model of Rumsey and Gatski $^{12}$ replaces the linear Boussinesq approximation with the following non-linear relationship

$$
\begin{array}{r}
-\rho \tau_{i j}=2 \mu_{t}^{*}\left\{S_{i j}+\left[a_{2} a_{4}\left(S_{i k} W_{k j}-W_{i k} S_{k j}\right)\right.\right. \\
\left.\left.-2 a_{3} a_{4}\left(S_{i k} S_{k j}-\frac{1}{3} S_{k l} S_{l k} \delta_{i j}\right)\right]\right\}-\frac{2}{3} \rho K \delta_{i j}
\end{array}
$$

where

$$
\mu_{t}^{*}=-\rho K \alpha_{1}
$$

The strain rate tensor is given in Equation 5, the rotation rate tensor is given by

$$
W_{i j}=\frac{1}{2}\left(\frac{\partial u_{i}}{\partial x_{j}}-\frac{\partial u_{j}}{\partial x_{i}}\right)
$$

and $\alpha_{1} / \tau(\tau \equiv 1 / \omega)$ is obtained from the solution of the cubic equation:

$$
\left(\frac{\alpha_{1}}{\tau}\right)^{3}+p\left(\frac{\alpha_{1}}{\tau}\right)^{2}+q\left(\frac{\alpha_{1}}{\tau}\right)+r=0
$$

See Rumsey and Gatski ${ }^{12}$ for the definition of $p, q, r$ and the solution of the above equation.
The following $K$ and $\omega$ transport equations are coupled with this non-linear stress-strain relationship:

$$
\begin{aligned}
& \frac{\partial}{\partial t}(\rho K)+\frac{\partial}{\partial x_{k}}\left(\rho u_{k} K\right)=\rho \mathcal{P}-f_{\beta^{*}} \rho \omega K+ \\
& \frac{\partial}{\partial x_{k}}\left[\left(\mu+\frac{\mu_{t}^{*}}{\sigma_{K}}\right) \frac{\partial K}{\partial x_{k}}\right](14) \\
& \frac{\partial}{\partial t}(\rho \omega)+\frac{\partial}{\partial x_{k}}\left(\rho u_{k} \omega\right)=\gamma \frac{\rho \omega}{K} \mathcal{P}-\beta \rho \omega^{2}+ \\
& \frac{\partial}{\partial x_{k}}\left[\left(\mu+\frac{\mu_{t}^{*}}{\sigma_{\omega}}\right) \frac{\partial \omega}{\partial x_{k}}\right]
\end{aligned}
$$

where the coefficients are updated as in Rumsey ${ }^{14} \sigma_{k}=$ $1, \sigma_{\omega}=\kappa^{2} /\left[\sqrt{C_{\mu}}(\beta-\gamma)\right], \kappa=0.41, \gamma=0.53$, $\beta=0.83$, and $C_{\mu}=0.0895$. The function $f_{\beta^{*}}$ is from Wilcox $^{15}$

$$
f_{\beta^{*}}= \begin{cases}1 & \text { if } \chi_{k} \leq 0 \\ \frac{1+680 \chi_{k}^{2}}{1+400 \chi_{k}^{2}} & \text { if } \chi_{k}>0\end{cases}
$$

where

$$
\chi_{k}=\frac{C_{\mu}^{2}}{\omega^{3}} \frac{\partial K}{\partial x_{j}} \frac{\partial \omega}{\partial x_{j}}
$$

\section{Computational Approach}

The CFD code ISAAC ${ }^{16}$ is used in this study. ISAAC is a second-order, upwind, finite-volume method where advection terms in the mean and turbulence equations are solved using Roe's approximate Riemann solver ${ }^{17}$ coupled with the MUSCL scheme. ${ }^{18}$ Viscous terms are calculated with a central difference approximation. Mean and turbulence equations are solved coupled using an implicit spatially split, diagonalized approximate factorization solver.

At the body surface, the no-slip boundary condition is imposed by setting the mean velocity component and the turbulent kinetic energy to zero, applying an adiabatic wall condition for the temperature, and setting the wall normal pressure gradient to zero. The wall condition for $\omega$ is due to Menter. ${ }^{19}$ At inflow and farfield boundaries, conditions based on solving a one-dimensional Riemann problem are imposed on all solution variables. Symmetry conditions are applied at the symmetry planes and zero gradient conditions are applied at the outflow plane.

\section{Results and Discussion}

Two turbulent flow fields, one incompressible and the other compressible, characterized by large separation and vortical flow regions are investigated. The incompressible case is the $6: 1$ prolate spheroid at high angleof-attack and high Reynolds number. The compressible case is the supersonic flow over an ogive cylinder. Both cases are predicted using the turbulence models described previously and results compared with experimental measurements. 


\section{Prolate Spheroid}

The prolate spheroid is a simple geometry that can provide significant understanding of the complex flow that can develop about slender bodies at incidence. An attached boundary layer is formed on the windward side of the prolate spheroid. The flow accelerates around the body until it separates due to the adverse pressure gradient. The flow then rolls up into a separated vortex inducing a secondary flow separation and vortex roll up between the primary vortex and the body.

As noted previously, a variety of wind tunnel tests have been carried out for a 6:1 prolate spheroid at DLR using a glass-fiber model of $4 \mathrm{~mm}$ wall thickness having a major axis of $2.4 \mathrm{~m}$ and a minor axis of $0.4 \mathrm{~m}$. Of interest here is the higher Reynolds number case tested at ONERA. The results presented here correspond to the $30^{\circ}$ angle of attack, $R e=43 \times 10^{6}$ case. This case has previously been used by an AGARD Working Group ${ }^{20}$ to test boundary layer solvers.

The experimental sting is modeled in the prolate spheroid calculations. On the surface of the spheroid, wall boundary conditions were imposed that specified zero velocity, adiabatic wall temperature, zero pressure gradient and zero turbulent kinetic energy. The calculations were made on a half body using symmetry boundary conditions. At the outflow plane, all variables were specified with zero gradient, and characteristic based boundary conditions were used on the outer boundary. The grid extended approximately seven body lengths upstream of the nose, six body lengths downstream of the end of the spheroid and approximately nine body lengths to the farfield. The experiment did not specify a transition location. Transition is specified computationally at $x / L \approx 0.02$, where $L$ is the body length.

A fine grid consisting of 129 points in the streamwise direction, 129 points in the wall normal direction and 73 points in the circumferential direction $(129 \times 129 \times 73)$ was generated using a two-dimensional hyperbolic grid generator and then rotating the grid about the axis of symmetry. A coarse grid $(65 \times 65 \times 37)$ was generated by eliminating alternate grid points in each coordinate direction. The coarse grid had a maximum $y^{+}$for the first point off the wall of approximately 0.67 with an average $y^{+}$for the first point off the wall of approximately 0.44 . A minimum of 25 points were located in the attached boundary layer regions. The fine grid had a maximum $y^{+}$for the first point off the wall of approximately 0.31 with an average $y^{+}$for the first point off the wall of approximately 0.21 . A minimum of 60 points were located in the attached boundary layer regions.

The solutions were computed at a Mach number of 0.25 . Other calculations were performed at nearby Mach numbers and showed no differences in the computed quantities. Solution residuals, surface pressures and skin friction were monitored to ensure iterative convergence.

Figure 1 shows the top and side view of the surface streamlines calculated on the fine grid with the $K-\omega$ model. The topology of the flow is easily seen in this figure. The primary separation line forms at $x / L \approx 0.1$ at the leeward symmetry plane and progresses windward down the body. The primary reattachment occurs in the plane of symmetry. Secondary separation and reattachment lines show the roll up of the secondary vortex between the primary vortex and the body.

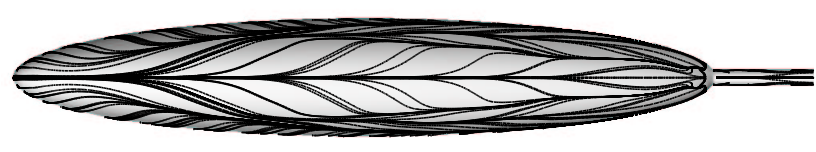

(a) Top View

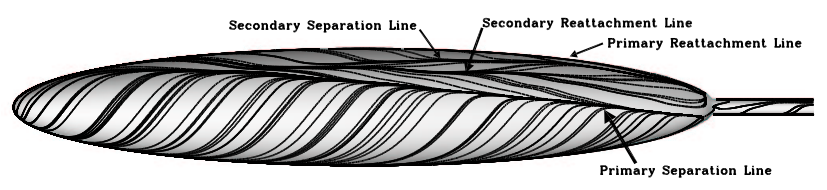

(b) Side View

Fig. 1 Surface streamlines for prolate spheroid at $30^{\circ}$ angle of attack calculated with the $K-\omega$ model on the fine grid.

Surface pressure coefficients calculated on the coarse and fine grids for three streamwise locations are plotted as a function of circumferential location $(\phi=0$ corresponds to the windward symmetry plane and $\phi=180$ corresponds to the leeward symmetry plane) in Figure 2 for the $K-\omega$ model and in Figure 3 for the EASM model. The solutions on the coarse and fine grids for both the $K-\omega$ and EASM models at the $x / L=0.09$ location indicate attached flow from the windward to the leeward side. This location is just upstream of the primary separation location shown in Figure 1. The solutions for both the $K-\omega$ and EASM model show very little change at the $x / L=0.09$ location with grid refinement.

The solutions at $x / L=0.51$ show a region of attached flow stretching from the windward plane to $\phi \approx 130^{\circ}$. The attached flow region for the $x / L=0.81$ location extends to $\phi \approx 110^{\circ}$. The coarse and fine grid solutions compare well with each other and the experimental data near the windward plane. However, as the separation location is approached, large variations arise in the coarse 
and fine solutions for both the $K-\omega$ and EASM models. The fine grid predicts an earlier separation than the coarse grid which is in better agreement with the experimental data. Additionally, the fine grid does a better job of predicting the pressure drop under the secondary vortex. Nevertheless, the variations in the pressure clearly indicate that the fine grid is still inadequate to resolve the flow.
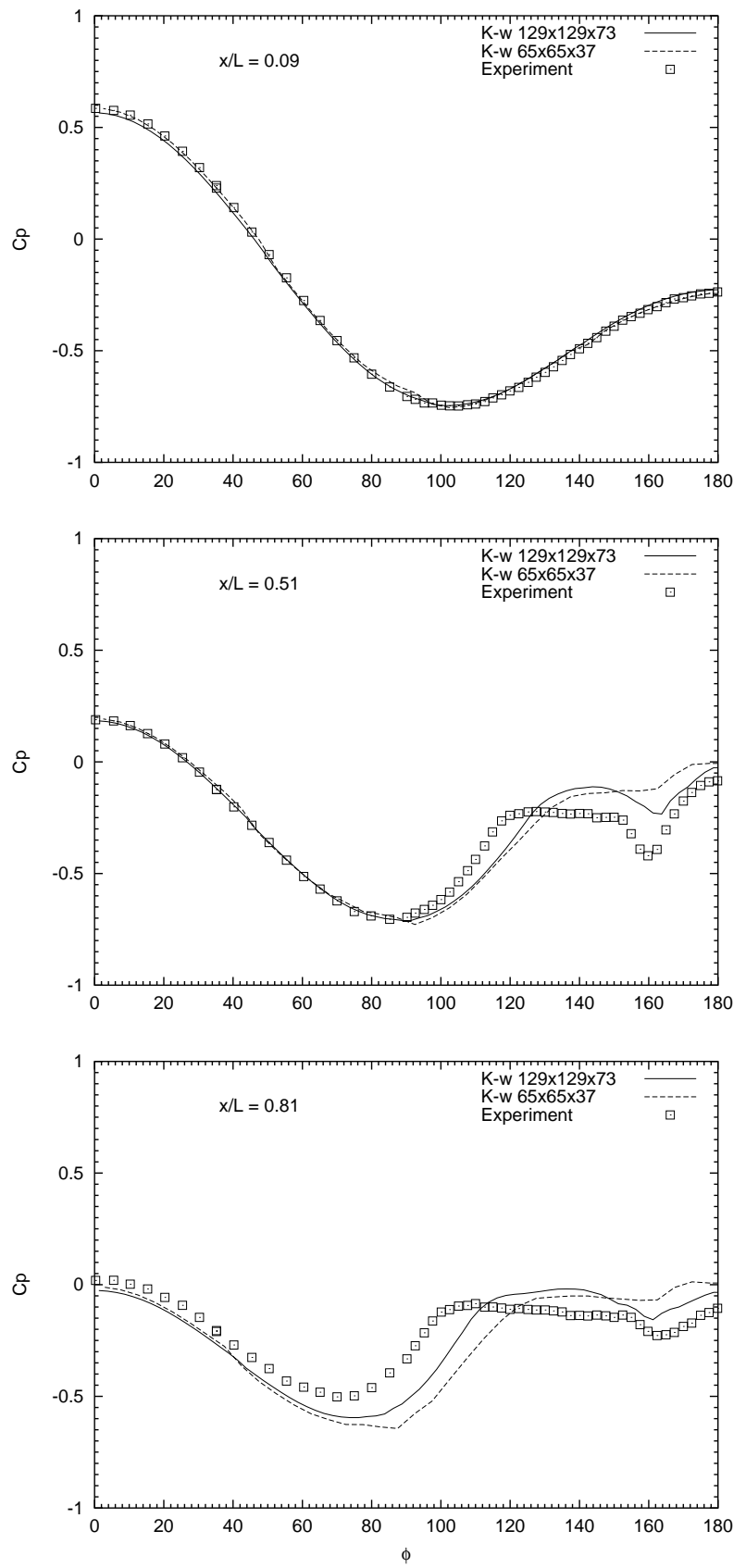

Fig. 2 Pressure coefficient for $K-\omega$ model on coarse and fine grid at $x / L=0.09,0.51$ and 0.81 .

The pressure coefficients calculated with the $K-\omega$ and the EASM model are shown in Figure 4 for the $129 \times$
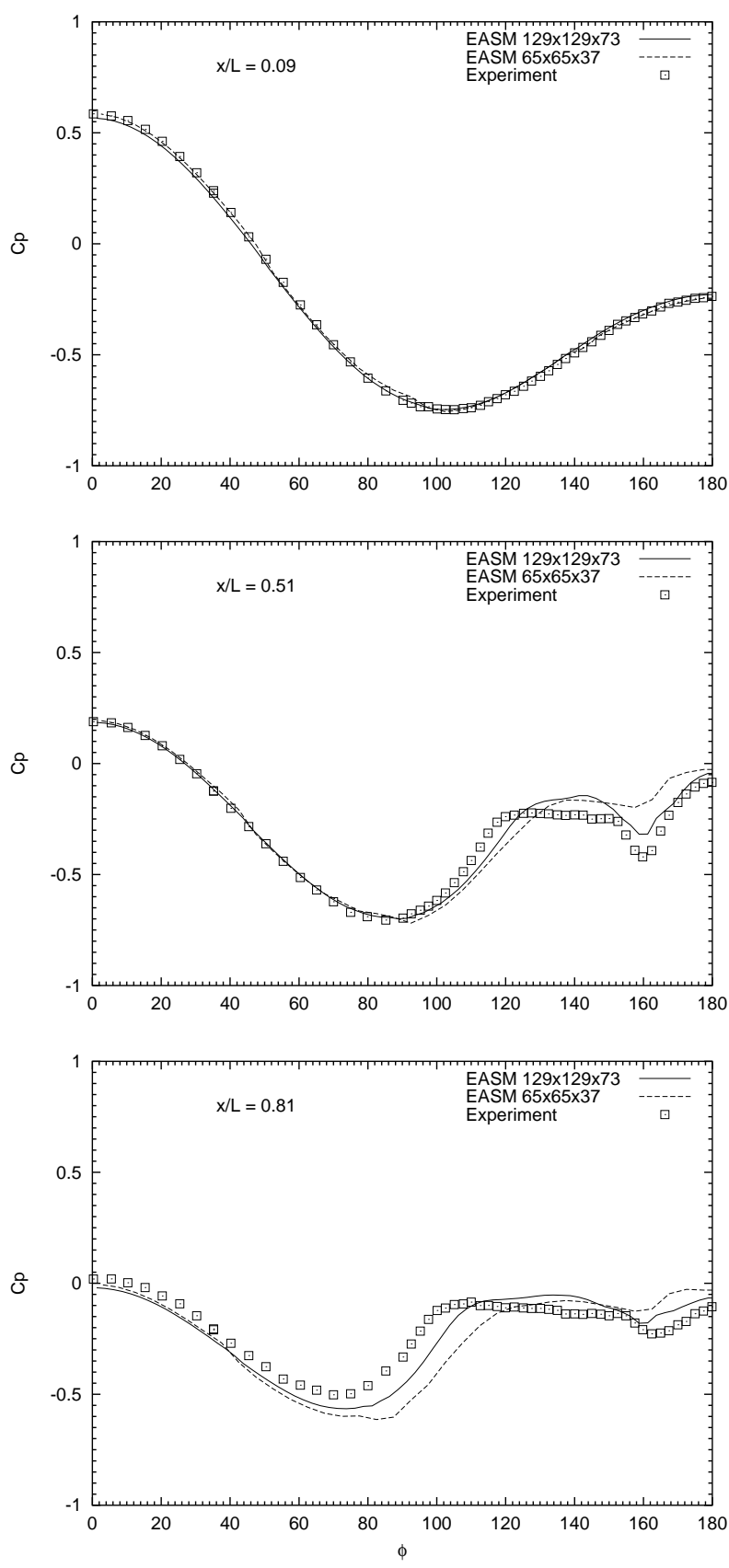

Fig. 3 Pressure coefficient for EASM model on coarse and fine grid at $\mathrm{x} / \mathrm{L}=0.09,0.51$ and 0.81 .

$129 \times 73$ grid. Both models perform well at the upstream $(x / L=0.09)$ plane which contains only attached flow. The two models are virtually indistinguishable from each other and match experimental pressures very well.

Both models predict the attached flow region at $x / L=$ 0.51 very well and agree well with each other. However, the EASM model separates earlier than the $K-\omega$ model and predicts a stronger pressure trough under the secondary vortex. The models agree very well with each other at $x / L=0.81$ only up to $\phi \approx 40^{\circ}$; well 
short of separation which occurs at $\phi \approx 110^{\circ}$ for the EASM model at this location. The pressures are consistently predicted lower than the experimental values at this streamwise location. The EASM model again predicts an earlier separation and a larger pressure trough under the secondary vortex. The EASM model predicts an earlier separation, a lower pressure plateau and a stronger pressure trough under the secondary vortex than the isotropic eddy viscosity model and more closely matches the experimental data.
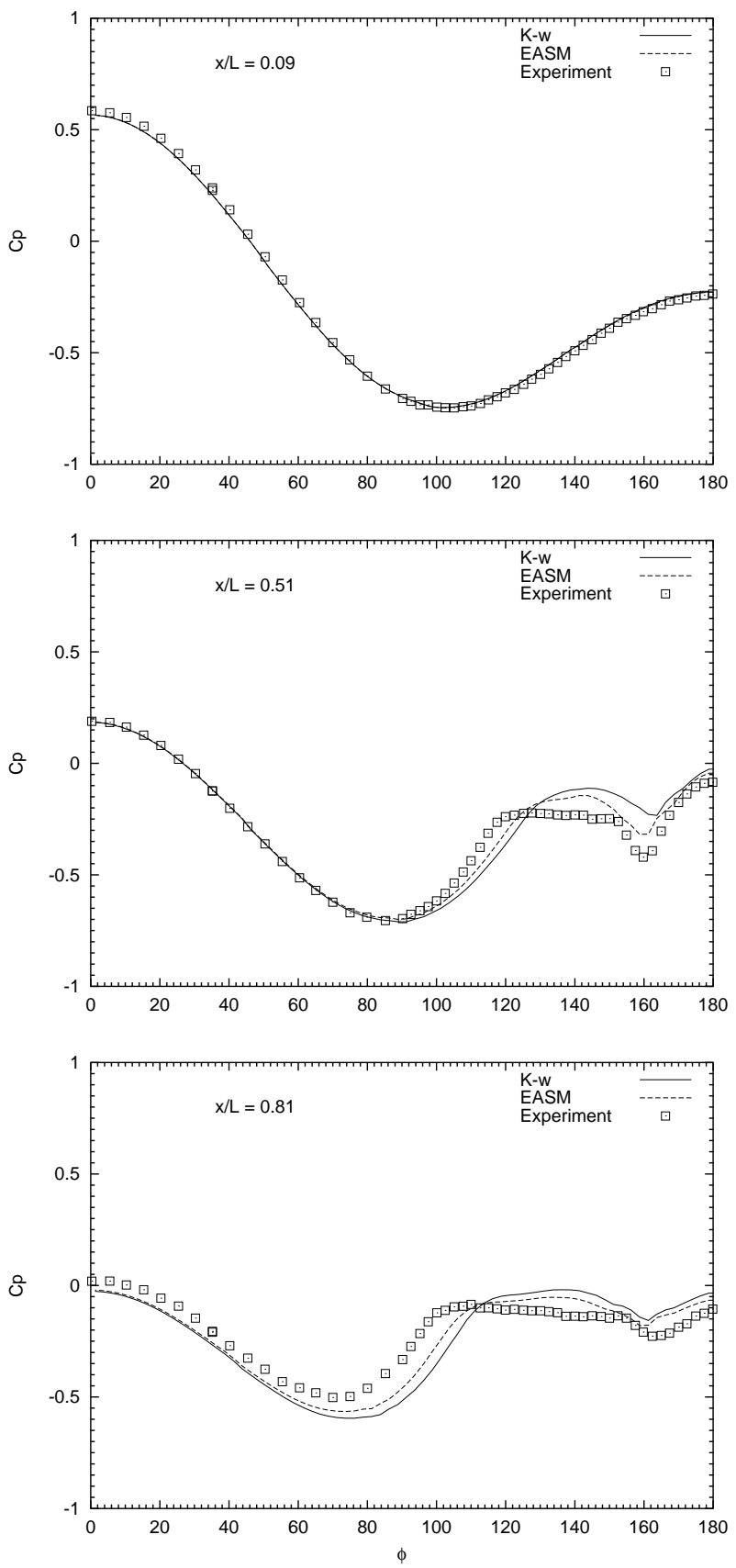

Fig. 4 Pressure coefficient for $K-\omega$ and EASM models at $\mathrm{x} / \mathrm{L}=\mathbf{0 . 0 9}, 0.51$ and 0.81 on fine grid.
The primary separation line is shown in Figure 5 for both models on both grids. This figure confirms the results shown in the pressure plots; the EASM model predicts earlier separation on each grid than the $K-\omega$ model predicts. Figure 5 also confirms that the solution on the fine grid has not achieved grid independence and a finer grid is required.

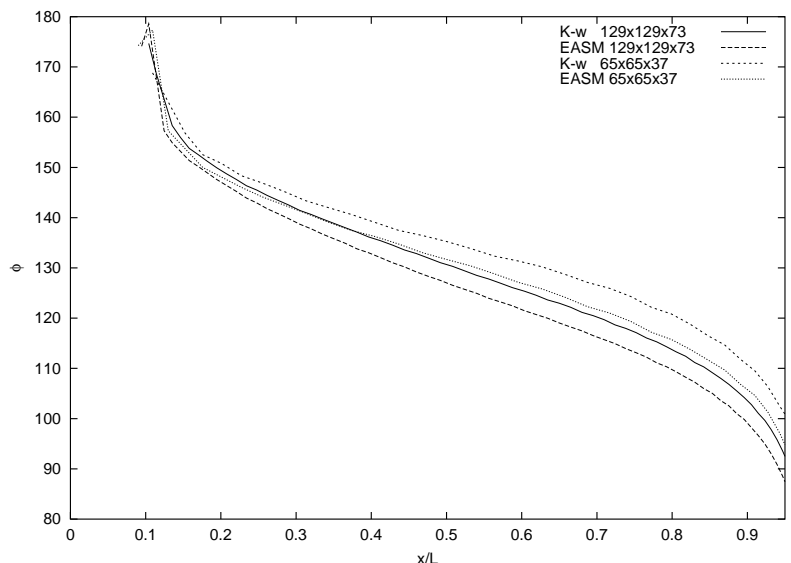

Fig. 5 Separation line location predictions for $K-\omega$ and EASM models on fine and coarse grid.

While the pressure predictions in the attached boundary layer regions of the prolate spheroid agree well between the two models and the experimental data, the details of the predicted boundary layer differ substantially. Figure 6 shows a typical profile of the turbulent kinetic energy at $x / L=0.524$ and $\phi=53.75^{\circ}$ calculated on the fine grid with each model ( $n$ is the distance normal to the surface). The EASM model clearly predicts a lower turbulent kinetic energy out to the edge of the boundary layer than the $K-\omega$ model. To further illustrate this point, the maximum turbulent kinetic energy in the boundary layer was calculated at each $\phi$ location at the same streamwise location and is shown in Figure 7. The minimum in the turbulent kinetic energy maximum correlates well with the separation location. The EASM model consistently predicts a lower maximum turbulent kinetic energy through the attached boundary layer region but then predicts a higher turbulence kinetic energy through the primary and secondary vortex.

Although the results presented represent a selfconsistent validation of two turbulence models for the flow over a prolate spheroid, it is apparent that the results still suffer from a lack of adequate resolution of the flow field. Further refinement in the grid structure needs to be undertaken in order to accurately predict the attached flow portion of this flowfield which is critical to accurately predicting the onset of separation.

\section{Supersonic Ogive Cylinder}

The supersonic flow about a 3-caliber tangent ogivecylinder at Mach 2.0 and angles of attack ranging from 


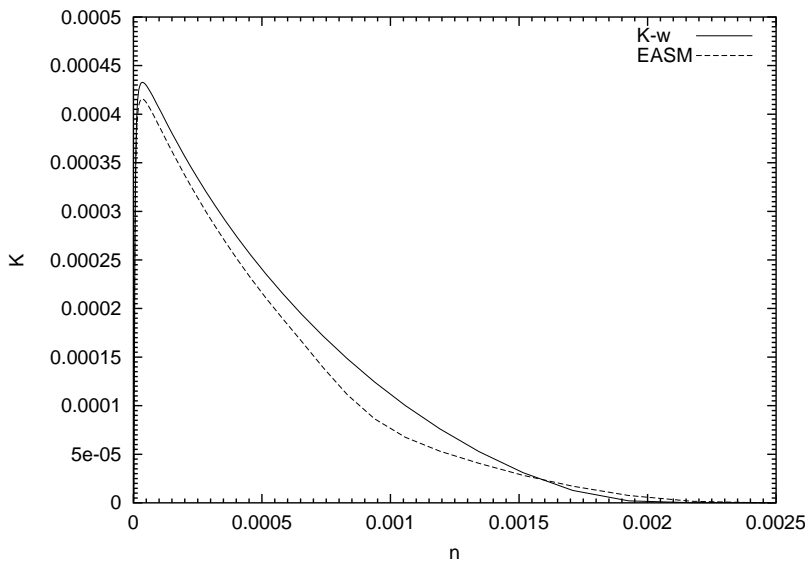

Fig. 6 Turbulent kinetic energy profiles for $K-\omega$ and EASM models at $x / L=0.524, \phi=53.75^{\circ}$.

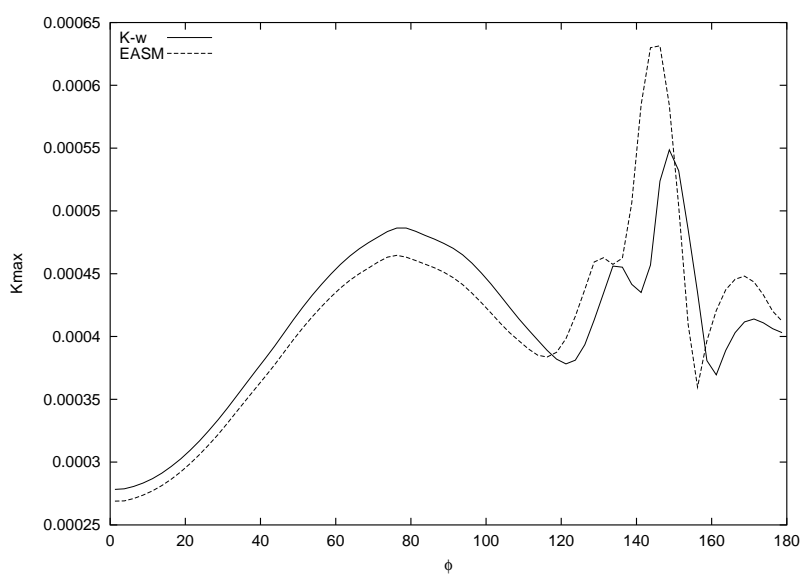

Fig. 7 Maximum turbulent kinetic energy for $K-\omega$ and EASM models at $x / L=0.524$.

$5^{\circ}$ to $20^{\circ}$ was investigated at ONERA. ${ }^{11}$ The Reynolds number based on the body length was $1.44 \times 10^{6}$. Experiments were performed for natural transition and transition fixed at $x / d=1$, where $d$ is the body diameter.

Grids were generated with a two-dimensional hyperbolic grid generator by rotating the grid about the axis of symmetry. In total, three grids were utilized. A coarse grid consisting of 67 points in the streamwise direction, 43 points in the wall normal direction and 37 points circumferentially $(67 \times 43 \times 37)$ and a medium grid consisting of 67 points in the streamwise direction, 85 points in the wall normal direction and 71 points circumferentially $(67 \times 85 \times 71)$. Note that the streamwise spacing was maintained in order to accurately capture the bow shock. An additional fine grid consisting of 99 points in the streamwise directions, 115 points normal to the wall and 101 points circumferentially was used to investigate grid convergence on the $\alpha=20^{\circ}$ case.

Two cases for the ogive cylinder were calculated; $\alpha=10^{\circ}$ and $\alpha=20^{\circ}$. The $10^{\circ}$ angle-of-attack case corresponds to moderately separated flow while the $20^{\circ}$ angle-of-attack case is characterized by a large separated region.

Inflow values of all flow quantities were held fixed at freestream values. Wall conditions were imposed with zero velocity, zero turbulent kinetic energy, adiabatic wall temperature and zero pressure gradient on the surface of the ogive cylinder. Outflow variables were set with a zero gradient condition. Symmetry conditions were imposed on the symmetry planes. Transition was specified at the experimental location, $x / d=1.0$.

The wall pressures predicted by the $K-\omega$ and EASM models on the $67 \times 85 \times 71$ grid are shown in Figure 8 for the $10^{\circ}$ angle-of-attack case at four streamwise locations. As in the prolate spheroid, $\phi$ is measured from the windward symmetry plane to the leeward symmetry plane. The agreement is good, especially at the downstream stations, where the secondary separation is reproduced well by the calculations. The $K-\omega$ model consistently predicts a lower pressure for the minimum pressure. The EASM model predicts a minimum pressure that is closer to the experimentally measured value. Additionally, the EASM model predicts a lower pressure under the primary vortex $\left(\phi \approx 155^{\circ}\right.$ at $x / d=7.5$ and 8.5$)$ that is in closer agreement to the experiment than the $K-\omega$ model.

Figure 9 shows the grid convergence of the EASM model for the $20^{\circ}$ angle-of-attack case. The separation location moves windward with increasing grid density and the pressure plateau in the separated region moves closer to the experimental value. The coarse grid is clearly insufficient to resolve this flow. However, there are small but still noticeable differences between the medium and fine grids especially in the separated region and under the primary and secondary vortices. Even with the finest grid resolution the solution is not grid converged.

The wall pressures predicted by the $K-\omega$ and EASM models on the $67 \times 85 \times 71$ grid are shown in Figure 10 for the $20^{\circ}$ angle-of-attack case. The agreement with data is very good except near $\phi=80^{\circ}$ where the flow separates. The EASM model again predicts earlier separation and a pressure level at separation that is closer to the experimentally measured value. The $K-\omega$ model more closely matches the experimental pressures on the leeward side of the ogive cylinder at the two upstream locations.

\section{Concluding Remarks}

Two flows with extensive cross-flow separation were investigated with the Reynolds averaged Navier-Stokes equations using a two-equation eddy-viscosity model and an explicit algebraic Reynolds stress model. The flows were the incompressible 6:1 prolate spheroid at $30^{\circ}$ angle of attack and the supersonic $(M=2)$ ogive cylinder at $10^{\circ}$ and $20^{\circ}$ angles of attack. It was found from grid convergence tests on both test cases that grid con- 

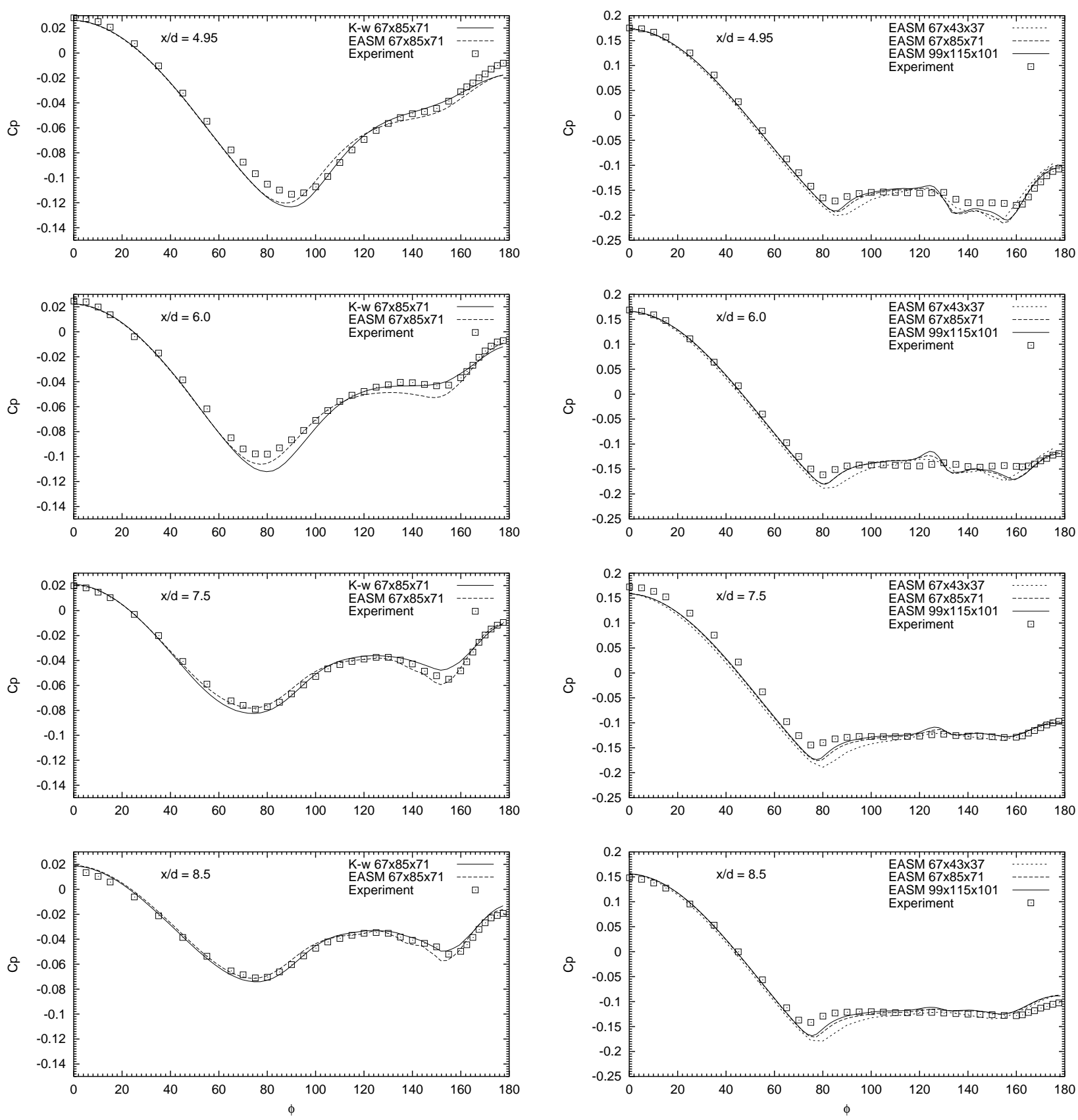

Fig. 8 Pressure coefficient on ogive cylinder at $10^{\circ}$ angle of attack for $K-\omega$ and EASM models at $\mathrm{x} / \mathrm{d}=4.95,6.0,7.5$ and 8.5.

vergence had not been achieved even with a 1.2 million node grid for the prolate spheroid. Additional computations on a refined grid are required to demonstrate grid convergence and to unambiguously evaluate the predictive capabilities of both models.

The $K-\omega$ model of Wilcox ${ }^{13}$ accurately predicted the surface pressures for the ogive cylinder at the lower angle of attack that had moderate separation. However, for the two cases with larger separations, the prolate spheroid at $30^{\circ}$ and the ogive cylinder at $20^{\circ}$, the $K-\omega$ model con-

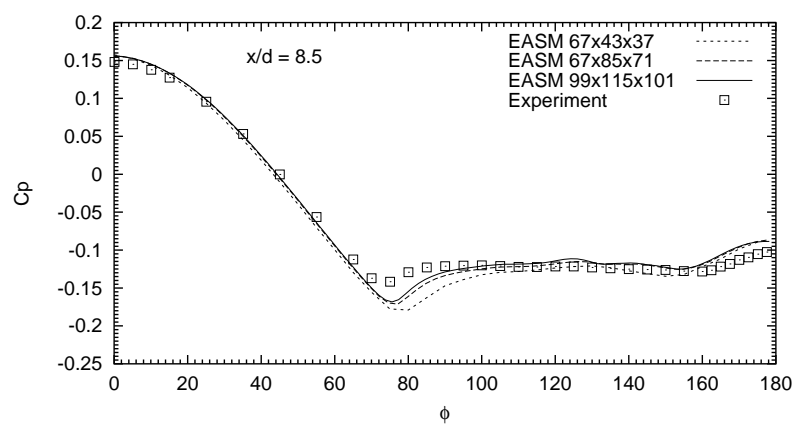

Fig. 9 Grid convergence of pressure coefficient on ogive cylinder at $20^{\circ}$ angle of attack for EASM model at $\mathbf{x} / \mathbf{d}=$ 4.95, 6.0, 7.5 and 8.5.

sistently predicted a smaller separation occurring later than the data showed. The EASM model of Rumsey and Gatski ${ }^{12}$ consistently predicted an earlier separation with a larger vortical flow region than the $K$ - $\omega$ linear eddy viscosity model, and is in closer agreement with the experimental data. Recall that one of the key advantages of the algebraic stress model is that it accounts for the effect of Reynolds stress anisotropy; whereas the isotropic eddy viscosity model does not. This causes the linear eddy viscosity model to be more dissipative than the EASM. 

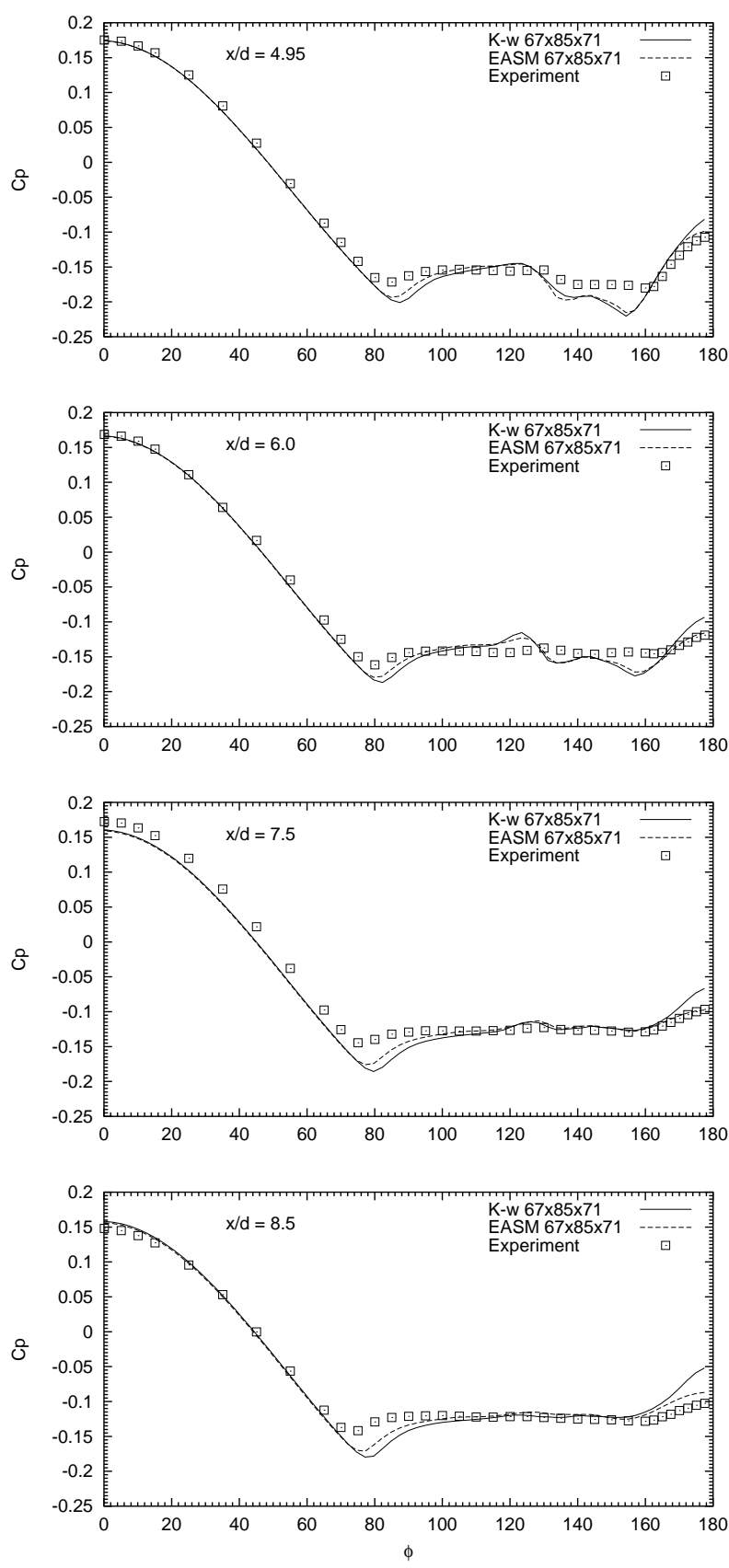

Fig. 10 Pressure coefficient on ogive cylinder at $20^{\circ}$ angle of attack for $K-\omega$ and EASM models at $\mathrm{x} / \mathrm{d}=\mathbf{4 . 9 5}, \mathbf{6 . 0}, 7.5$ and 8.5 on medium grid.

The results obtained here, which generally show the improved predictive capability of the EASM, suggest that this better accounting of the turbulent stress anisotropies more accurately predicts the effect of the turbulence on the embedded vortical regions that characterize both the incompressible prolate spheroid and the supersonic ogive cylinder flows. Future studies into more accurately assessing and quantifying these anisotropic effects appear warranted.

\section{Acknowledgements}

The authors gratefully acknowledge the support of the NATO RTO under project G107 "Turbulence Modeling for Flows with Extensive Cross Flow Separation" under an agreement between the Hellenic Air Force Academy and the NASA Langley Research Center.

\section{References}

${ }^{1}$ Panaras, A. G., "On the calculation of the turbulent incompressible flow about a 6:1 prolate spheroid at high incidence," Chapter 11 in Flows at Large Reynolds Numbers, Ed. H. Schmitt, Computational Mechanics Publications, 1997.

${ }^{2}$ Panaras, A. G., "Numerical investigation of the high-speed conical flow past a sharp fin," J. Fluid Mech., Vol. 236, pp. 607-633, 1992.

${ }^{3}$ Panaras, A. G., "The effect of the structure of swept shockwave/turbulent boundary-layer interactions on turbulence modelling," J. Fluid Mech., Vol. 338, pp. 203-230, 1997.

${ }^{4}$ Panaras, A. G., "Algebraic Turbulence Modelling for Swept Shock-Wave/Turbulent Boundary-Layer Interactions," AIAA Journal, Vol. 35, No. 3, pp. 456-463, 1997.

${ }^{5}$ Panaras, A.G., "Calculation of Flows Characterized by Extensive Cross Flow Separation," Proc. 4th Europ. Symp. Aerothermodynamics for Space Applications, 2001, Capua, Italy, ESA SP-487, March 2002, pp.349-359.

${ }^{6}$ Ahn, S. and Simpson, R.L., "Cross-Flow Separation on a Prolate Spheroid at Angles of Attack," AIAA Paper 92-0428, January 1992.

${ }^{7}$ Chesnakas, C.J. and Simpson, R.L., "Measurements of the Turbulence Structure in the Vicinity of a 3-D Separation," J. Fluids Eng., Vol. 118, June 1996, pp. 268-275.

${ }^{8}$ Tsai, C-Y. and Whitney, A. K., "Numerical Study of ThreeDimensional Flow Separation for a 6:1 Ellipsoid," AIAA Paper 990172, January, 1999.

${ }^{9}$ Kim, S.E., Rhee, S.H., and Cokljat, D., "Application of Modern Turbulence Models to Vortical Flow Around a 6:1 Prolate Spheroid at Incidence," AIAA Paper 2003-0429, January 2003.

${ }^{10}$ Constantinescu, G. S., Pasinato, H., Wang, Y-Q., and Squires, K. D., "Numerical Investigation of Flow Past a Prolate Spheroid," AIAA Paper 2002-0588, January, 2002.

${ }^{11}$ Barberis, D., "Supersonic vortex flow around a missile body," AGARD AR-303, Vol. II, 1994.

${ }^{12}$ Rumsey, C.L. and Gatski, T.B., "Recent Turbulence Model Advances Applied to Multielement Airfoil Computations," AIAA Paper 2000-4323, August 2000.

${ }^{13}$ Wilcox, D. C., "Reassessment of the Scale Determining Equation for Advanced Turbulence Models," AIAA Journal, Vol. 26, No. 11, November 1988.

${ }^{14}$ Rumsey, C.L., "Effect of Turbulence Models on Two MassivelySeparated Benchmark Flow Cases," NASA TM 2003-212412, May 2003.

${ }^{15}$ Wilcox, D. C., Turbulence Modeling for CFD, 2nd Ed., DCW Industries, La Canada, 1998.

${ }^{16}$ Morrison, J. H., "A Compressible Navier-Stokes Solver with Two-Equation and Reynolds Stress Turbulence Closure Models," NASA CR-4440, May 1992.

${ }^{17}$ Roe, P. L., "Approximate Riemann Solvers, Parameter Vectors, and Difference Schemes," Journal of Computational Physics, Vol. 43, pp. 357-372, 1981.

${ }^{18}$ van Leer, B., "Towards the Ultimate Conservative Difference Schemes V. A Second Order Sequel to Godunov's Method," J. Comp. Physics, Vol. 32, pp. 101-136,1979.

${ }^{19}$ Menter, F.R., "Improved Two-Equation $K-\omega$ Turbulence Models for Aerodynamic Flows,” NASA TM 103975, Oct. 1992. 
AIAA-2003-3532

${ }^{20}$ Meier, H. U. and Kreplin, H.-P., "Description of the DFVLR prolate spheroid and its instrumentation," in Calculation of $3 D$ Separate Turbulent Flows in Boundary Layer Limit, AGARD AR-255, 1990. 